\title{
Spontaneous Anterograde Flow of the Infarct Artery Preserves Myocardial Perfusion and Fatty Acid Metabolism in Patients With Anterior Acute Myocardial Infarction
}

\author{
Satoshi Kurisu, MD; Ichiro Inoue, MD; Takuji Kawagoe, MD; Masaharu Ishihara, MD; \\ Yuji Shimatani, MD; Naoya Mitsuba, MD; Takaki Hata, MD; Yasuharu Nakama, MD; \\ Tomohiko Kisaka, MD; Yasufumi Kijima, MD
}

\begin{abstract}
Background It remains unclear whether spontaneous anterograde flow preserves myocardial fatty acid metabolism in patients with acute myocardial infarction (AMI).

Methods and Results The present study comprised 129 patients with a first anterior AMI in whom Thrombolysis in Myocardial Infarction (TIMI) 3 flow was obtained on the final angiogram: 28 patients with spontaneous anterograde flow and 101 patients with total occlusion on the initial angiogram. Thallium-201 (201 $\mathrm{Tl})$ and iodine123 - $\beta$-methyl-p-iodophenyl penta-decanoic acid ( ${ }^{123}$ I-BMIPP) dual-isotope myocardial single-photon emission computed tomography (SPECT) was performed at $6.5 \pm 4.2$ days after onset. The SPECT image was divided into 17 segments, and each segment was graded with scores between 0 and 4 ( 0 , normal uptake; 4 , defective). The sum of each score was defined as the total defect score (TDS). TDS values for ${ }^{201}{ }^{\mathrm{Tl}}(9.0 \pm 7.4$ vs $16.8 \pm 12.2$, $\mathrm{p}<0.01)$ and ${ }^{123}$ I-BMIPP $(19.3 \pm 11.6$ vs $24.1 \pm 10.4, \mathrm{p}<0.05)$ were significantly lower in patients with spontaneous anterograde flow than in those with total occlusion.

Conclusions These results suggest that spontaneous anterograde flow of the infarct artery preserves not only myocardial perfusion but also fatty acid metabolism in patients with AMI. ${ }^{23}$ I-BMIPP SPECT image may underestimate the area at risk especially in patients with spontaneous anterograde flow. (Circ J 2005; 69: 427-431)
\end{abstract}

Key Words: BMIPP; Reperfusion; Ventricular function

$\mathbf{P}$ revious studies demonstrated that spontaneous anterograde flow of the infarct artery was associated with better left ventricular function and more favorable outcome compared with total occlusion in patients with acute myocardial infarction (AMI) ${ }^{1-3}$ Although these beneficial effects of spontaneous anterograde flow are mainly because of reduced infarct size, it remains unclear how spontaneous anterograde flow affects myocardial metabolism in patients with AMI.

Iodine-123- $\beta$-methyl-p-iodophenyl penta-decanoic acid (123I-BMIPP) is a tracer for the evaluation of coronary artery disease, and identifies altered fatty acid metabolism after reperfusion in AMI-6 In the present study we used thallium-201 $\left({ }^{201} \mathrm{Tl}\right)$ and ${ }^{123}$ I-BMIPP to test the hypothesis that spontaneous anterograde flow could preserve not only myocardial perfusion but also fatty acid metabolism in patients with anterior AMI. We also compared the effect of spontaneous anterograde flow and very early reperfusion on myocardial perfusion and fatty acid metabolism.

(Received October 25, 2004; revised manuscript received January 17, 2005; accepted January 28, 2005)

Department of Cardiology, Hiroshima City Hospital, Hiroshima, Japan

Mailing address: Satoshi Kurisu, MD, Department of Cardiology, Hiroshima City Hospital, 7-33 Moto-machi, Naka-ku, Hiroshima 730-8518, Japan. E-mail: skurisu@nifty.com

\section{Methods}

Study Patients

The study group comprised 129 patients in Hiroshima City Hospital with a first anterior AMI in whom coronary angiography was performed within $12 \mathrm{~h}$ after the onset of chest pain, and in whom Thrombolysis in Myocardial Infarction (TIMI) 3 flow $^{7}$ was obtained on the final angiogram. AMI was diagnosed by chest pain consistent with ongoing myocardial ischemia for $>30 \mathrm{~min}$ and elevation of serum creatine kinase to more than twice the normal upper limit. To assess infarct size, serum creatine kinase was measured every $3 \mathrm{~h}$ for at least $24 \mathrm{~h}$ to obtain the peak value. Patients with signs of re-infarction were excluded. The study protocol was approved by the institutional ethics committee and written informed consent was obtained from all patients.

There were 28 patients with TIMI 2 or 3 flow of the infarct artery on the initial angiogram. The remaining 101 patients with TIMI 0 or 1 flow were divided into 2 groups: 42 patients in whom time to reperfusion was within $2 \mathrm{~h}$ (very early reperfusion) and 59 patients in whom time to reperfusion was more than $2 \mathrm{~h}$ (late reperfusion)?

\section{Electrocardiogram}

Electrocardiogram was obtained on admission routinely in all patients. ST elevation AMI or non-ST elevation AMI was diagnosed by the presence or the absence of ST elevation more than at least $2 \mathrm{~mm}$ in 2 or more contiguous leads. 


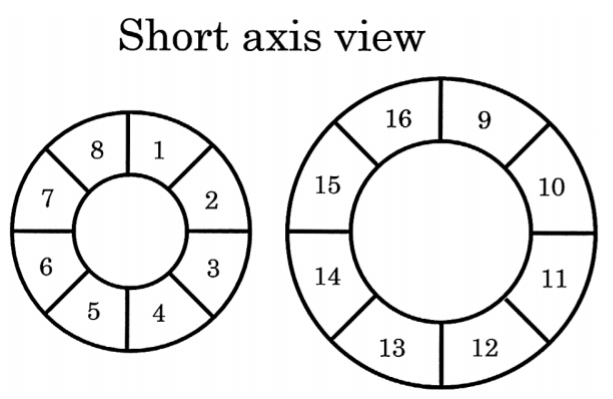

\section{Vertical long axis view}

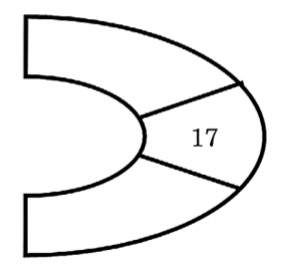

Fig 1. SPECT image of the left ventricle was divided into 17 segments for semiquantitative analysis. Short-axis slices were separated into 8 segments at the basal and midventricular levels. The apical portion of one segment was evaluated on vertical long-axis slices. Each segment was graded visually with scores between 0 and 4 ( 0 , normal uptake; 1 , mildly reduced; 2 , moderately reduced; 3 , severely reduced; 4 , defective), and the sum of each score was defined as the total defect score.

Table 1 Baseline Characteristics of the Study Patients

\begin{tabular}{|c|c|c|c|}
\hline & Spontaneous anterograde flow & Total occlusion & $p$-value \\
\hline No. of patients & 28 & 101 & \\
\hline Age (years) & $64 \pm 14$ & $60 \pm 11$ & NS \\
\hline Male gender & $18(64 \%)$ & $86(85 \%)$ & $<0.05$ \\
\hline Diabetes & $4(14 \%)$ & $20(20 \%)$ & $N S$ \\
\hline Hypertension & $11(39 \%)$ & $38(38 \%)$ & $N S$ \\
\hline Current smoker & $12(43 \%)$ & $62(61 \%)$ & NS \\
\hline Prodromal angina & $12(43 \%)$ & $34(34 \%)$ & $N S$ \\
\hline Electrocardiogram & & & $N S$ \\
\hline ST elevation AMI & $20(71 \%)$ & $87(86 \%)$ & \\
\hline Non-ST elevation AMI & $8(29 \%)$ & $14(14 \%)$ & \\
\hline Time to angiography $(h)$ & $4.3 \pm 3.1$ & $3.2 \pm 2.0$ & $<0.05$ \\
\hline Culprit lesion location & & & NS \\
\hline Proximal to first septal branch & $18(64 \%)$ & $60(59 \%)$ & \\
\hline Distal to first septal branch & $10(36 \%)$ & $41(41 \%)$ & \\
\hline Multivessel disease & $11(39 \%)$ & $29(29 \%)$ & NS \\
\hline Collateral circulation & $3(11 \%)$ & $40(40 \%)$ & $<0.01$ \\
\hline Reperfusion therapy & & & $N S$ \\
\hline Thrombolysis only & $1(4 \%)$ & $1(1 \%)$ & \\
\hline Coronary intervention & $26(92 \%)$ & $100(99 \%)$ & \\
\hline Neither & $1(4 \%)$ & $0(0 \%)$ & \\
\hline Peak creatine kinase $(I U / L)$ & $2,129 \pm 1,507$ & $4,302 \pm 2,553$ & $<0.01$ \\
\hline
\end{tabular}

\section{Cardiac Catheterization}

Emergency cardiac catheterization was performed via the right femoral artery or left brachial artery after heparin administration. Coronary angiography was performed in multiple projections before reperfusion therapy. Immediately after diagnostic angiography, reperfusion was performed with percutaneous coronary intervention or coronary thrombolysis, if indicated. The allocation of reperfusion therapy was not randomized and was based on the physician's decision.

\section{Angiographic Analysis}

All coronary angiograms were reviewed by 2 cardiologists unaware of the clinical data. Initial TIMI flow grade was assessed on the initial image, and final TIMI flow grade was assessed on the final coronary angiogram. Spontaneous anterograde flow was defined as TIMI 2 or 3 flow, and total occlusion was defined as TIMI 0 or 1 flow on the initial coronary angiogram. Multivessel coronary disease was defined as $\geq 75 \%$ stenosis remote from the infarct-related artery. Collateral circulation was considered present if partial or complete filling of the infarct artery distal to the culprit lesion was present. Predischarge angiography was performed approximately 14 days later. Left ventricular ejection fraction (LVEF) was calculated by the area-length method.

\section{${ }^{201}$ Tl and ${ }^{123}$ I-BMIPP Myocardial Single-Photon Emission Computed Tomography (SPECT)}

Resting ${ }^{201} \mathrm{Tl}$ and ${ }^{123} \mathrm{I}-\mathrm{BMIPP}$ dual-isotope myocardial
SPECT were performed approximately 6 days after the onset. After overnight fasting, an intravenous bolus injection of ${ }^{201} \mathrm{Tl}(111 \mathrm{MBq})$ and ${ }^{123}$ I-BMIPP $(111 \mathrm{MBq})$ was performed at rest and data acquisition was started $20 \mathrm{~min}$ later using a 3-headed SPECT system with low-energy, allpurpose parallel-hole collimators. A total of 60 projection images was obtained in a $128 \times 128$ matrix over 360 degrees, with 30 s/view. After reducing the matrix to $64 \times 64$, tomographic images along the vertical long, horizontal long and short axes were created with a Shepp and Logan filter. The SPECT image of the left ventricle was divided into 17 segments for semiquantitative analysis? Short-axis slices were separated into 8 segments at the basal and midventricular levels. The apical portion of one segment was evaluated on vertical long-axis slices (Fig 1). Each segment was graded visually with scores between 0 and 4 (0, normal uptake; 1 , mildly reduced; 2 , moderately reduced; 3 , severely reduced; 4 , defective) in a blinded manner by the 2 experienced cardiologists. The sum of each score was defined as the total defect score (TDS), reflecting the severity of impaired myocardial perfusion or impaired fatty acid metabolism. The TDS values for ${ }^{201} \mathrm{Tl}$ and ${ }^{123} \mathrm{I}-\mathrm{BMIPP}$ were compared, and the relation between ${ }^{201} \mathrm{Tl}$ and ${ }^{123} \mathrm{I}-$ BMIPP was defined as mismatch when the 123I-BMIPP TDS exceeded that for ${ }^{201} \mathrm{Tl}$ by 10 .

\section{Data Analysis}

Statistical analysis was performed with the chi-square test for categorical variables. The unpaired Student's t-test was used for continuous variables. Differences were con- 
Table 2 Baseline Characteristics of the Patients With Total Occlusion

\begin{tabular}{lccc}
\hline \hline & Very early reperfusion & Late reperfusion & p-value \\
\hline No. of patients & 42 & 59 & \\
Age (years) & $57 \pm 11$ & $62 \pm 11$ & $<0.01$ \\
Male gender & $39(93 \%)$ & $47(80 \%)$ & $N S$ \\
Diabetes & $8(19 \%)$ & $12(20 \%)$ & $N S$ \\
Hypertension & $14(33 \%)$ & $24(41 \%)$ & $N S$ \\
Current smoker & $26(62 \%)$ & $36(61 \%)$ & $N S$ \\
Prodromal angina & $7(17 \%)$ & $27(46 \%)$ & $<.01$ \\
Electrocardiogram & & & $N S$ \\
ST elevation AMI & $37(88 \%)$ & $50(85 \%)$ & \\
Non-ST elevation AMI & $5(12 \%)$ & $9(15 \%)$ & $<.01$ \\
Time to angiography $(h)$ & $1.7 \pm 0.4$ & $4.2 \pm 2.0$ & \\
Culprit lesion location & $30(71 \%)$ & $30(51 \%)$ & \\
Proximal to first septal branch & $12(29 \%)$ & $29(49 \%)$ & $N S$ \\
Distal to first septal branch & $9(21 \%)$ & $20(34 \%)$ & $N S$ \\
Multivessel disease & $19(45 \%)$ & $21(36 \%)$ & $N S$ \\
Collateral circulation & $1(2 \%)$ & $0(0 \%)$ & \\
Reperfusion therapy & $41(98 \%)$ & $59(100 \%)$ & \\
Thrombolysis only & $0(0 \%)$ & $0(0 \%)$ & \\
Coronary intervention & $4,249 \pm 2,198$ & $4,340 \pm 2,791$ & \\
$\quad$ Neither & & & \\
Peak creatine kinase (IU/L) & & \\
\hline
\end{tabular}
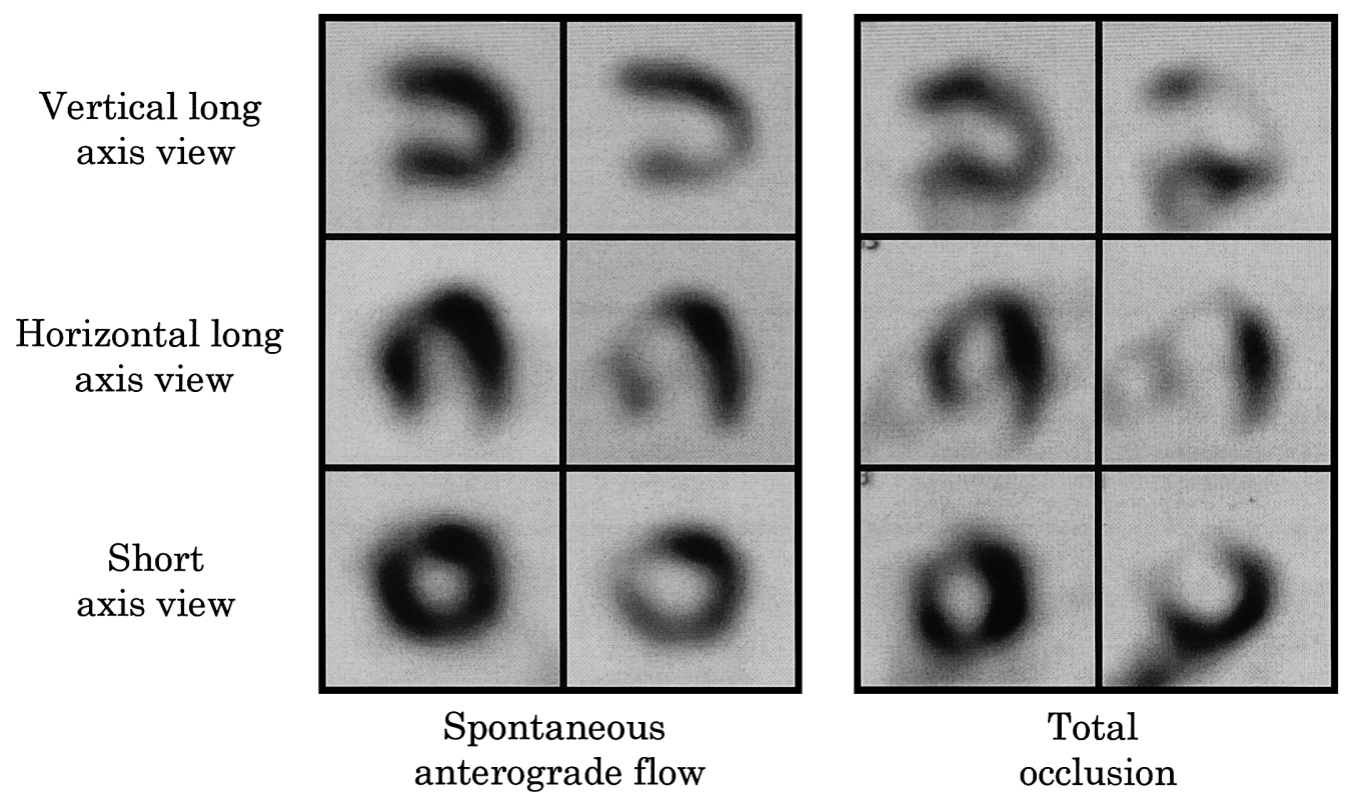

Fig 2. Representative resting ${ }^{201} \mathrm{Tl}$ (Left) and ${ }^{123}$ I-BMIPP (Right) myocardial SPECT images in patients with spontaneous anterograde flow and total occlusion who underwent reperfusion therapy. In a patient with spontaneous anterograde flow, the myocardial uptake of ${ }^{123}$ I-BMIPP was moderately reduced in the apical and anteroseptal segments, whereas that of ${ }^{201} \mathrm{Tl}$ was only mildly reduced. In a patient with total occlusion, the myocardial uptake of ${ }^{123}$ I-BMIPP was severely reduced in the apical and anteroseptal segments, whereas that of ${ }^{201} \mathrm{Tl}$ was moderately reduced.

sidered significant if the $\mathrm{p}$-value was $<0.05$. All data are expressed mean $\pm \mathrm{SD}$.

\section{Results}

\section{Baseline Characteristics}

There were no significant differences in the baseline characteristics between patients with spontaneous anterograde flow and those with total occlusion, except for more males, longer time to angiography and less collateral circulation in those with spontaneous anterograde flow (Table 1). The baseline characteristics of the patients with very early reperfusion and those with late reperfusion are shown in Table 2.

\section{Infarct Size}

The peak creatine kinase value was obtained in all patients and it was significantly lower in patients with spontaneous anterograde flow than in those with total occlusion $(2,129 \pm 1,507$ vs $4,302 \pm 2,553 \mathrm{IU} / \mathrm{L}, \mathrm{p}<0.01)$, even when compared with only those with very early reperfusion $(2,129 \pm 1,507$ vs $4,249 \pm 2,198 \mathrm{IU} / \mathrm{L}, \mathrm{p}<0.01)$.

\section{SPECT (Fig 2, Table 3)}

The TDS value for ${ }^{201} \mathrm{Tl}$ was significantly lower in patients with spontaneous anterograde flow than in those with 


\begin{tabular}{lccc}
\hline \hline & Spontaneous anterograde flow & Total occlusion & p-value \\
\hline TDS value for ${ }^{201}$ Tl & $9.0 \pm 7.4$ & $16.8 \pm 12.2$ & $<0.01$ \\
TDS value for ${ }^{223}$ I-BMIPP & $19.3 \pm 11.6$ & $24.1 \pm 10.4$ & $<0.05$ \\
Mismatch between ${ }^{201}$ Tl and ${ }^{123}$ I-BMIPP & $19(68 \%)$ & $42(42 \%)$ & $<0.05$ \\
\hline
\end{tabular}

total occlusion $(9.0 \pm 7.4$ vs $16.8 \pm 12.2, \mathrm{p}<0.01)$, even when compared with only those with very early reperfusion (9.0 \pm 7.4 vs $16.6 \pm 12.5, \mathrm{p}<0.01)$. The TDS value for ${ }^{123} \mathrm{I}-$ BMIPP was significantly lower in patients with spontaneous anterograde flow than in those with total occlusion $(19.3 \pm 11.6$ vs $24.1 \pm 10.4, p<0.05)$. There was a trend to lower TDS values for ${ }^{123}$ I-BMIPP in patients with spontaneous anterograde flow, even when compared to only those with very early reperfusion $(19.3 \pm 11.6$ vs $24.1 \pm 11.3$, $\mathrm{p}=0.10$ ). Mismatch between ${ }^{201} \mathrm{Tl}$ and ${ }^{123} \mathrm{I}-\mathrm{BMIPP}$ was observed more frequently in patients with spontaneous anterograde flow than in those with total occlusion $(68 \%$ vs $42 \%, \mathrm{p}<0.05)$, even when compared to only those with very early reperfusion ( $68 \%$ vs $40 \%, \mathrm{p}<0.05)$.

Predischarge angiography was performed in 100 patients $(78 \%)$ at $12 \pm 4$ days after the onset. All patients had a patent infarct artery. Both acute and predischarge left ventriculograms were obtained in 85 patients $(66 \%)$. The change in LVEF was significantly higher in patients with spontaneous anterograde flow than in those with total occlusion $(13 \pm 9 \%$ vs $3 \pm 16 \%, \mathrm{p}<0.05)$. There was a trend to a higher LVEF in patients with spontaneous anterograde flow, even when compared to only those with very early reperfusion $(13 \pm 9 \%$ vs $6 \pm 17 \%, p=0.16)$. Predischarge LVEF significantly correlated with the TDS values for ${ }^{201} \mathrm{Tl}$ $\left(\mathrm{r}^{2}=0.27, \mathrm{p}<0.01, \mathrm{n}=100\right)$ and 123I-BMIPP $\left(\mathrm{r}^{2}=0.28, \mathrm{p}<0.01\right.$, $\mathrm{n}=100)$.

\section{Discussion}

\section{Present Study}

By using ${ }^{201} \mathrm{Tl}$ and ${ }^{123} \mathrm{I}-\mathrm{BMIPP}$ dual-isotope myocardial SPECT, this study demonstrated that (1) spontaneous anterograde flow of the infarct artery preserved not only myocardial perfusion, but also fatty acid metabolism in patients with anterior AMI, and (2) these beneficial effects of spontaneous anterograde flow was superior even to those of very early reperfusion.

\section{Previous Studies}

Previous studies demonstrated the association between spontaneous anterograde flow and left ventricular function in AMI ${ }^{-3}$ Stone et al reported that patients with initial TIMI 3 flow before reperfusion therapy had better acute LVEF than those without? We also demonstrated that initial TIMI 3 flow was associated with better acute LVEF, but the acute LVEF of patients with initial TIMI 2 flow was similar to that of patients with initial TIMI 0 or 1 flow. On the other hand, compared with patients with TIMI 0 or 1 flow, the predischarge LVEF was significantly better not only in patients with initial TIMI 3 flow but also in patients with initial TIMI 2 flow? Thus, spontaneous anterograde flow seemed to preserve left ventricular function during the early phase of AMI. In the current study, we demonstrated that the TDS value for ${ }^{201} \mathrm{Tl}$ and peak creatine kinase value were significantly lower in patients with spontaneous anterograde flow than in those with total occlusion, suggesting that the beneficial effects of spontaneous anterograde flow resulted from a reduction in the infarct size.

\section{Myocardial Metabolism}

The major component of ${ }^{123}$ I-BMIPP in the myocardium is the triglyceride pool, and ${ }^{123}$ I-BMIPP is partially metabolized first by $\alpha$-oxidation, then by $\beta$-oxidation. Because the myocardial accumulation of ${ }^{123}$ I-BMIPP is associated with triglyceride synthesis, which in part reflects fatty acid utilization, ${ }^{123}$ I-BMIPP is used as a tracer for myocardial fatty acid metabolism. Matsunari et al reported that segments with reduced uptake of ${ }^{123} \mathrm{I}$-BMIPP showed ${ }^{201} \mathrm{Tl}$ redistribution on exercise redistribution ${ }^{201} \mathrm{Tl}$ imaging? Kawamoto et al reported that myocardial ischemia in which the uptake of 123I-BMIPP was more reduced than that of ${ }^{201} \mathrm{Tl}$ showed increased uptake of fluorodeoxyglucose! ${ }^{10}$ Those results indicate that reduced uptake of 123I-BMIPP represents myocardial ischemia. Moreover, previous studies showed that the degree of reduced uptake of 123I-BMIPP correlated with either the severity of coronary artery stenosis or the severity of ischemic attack in patients with angina pectoris! ${ }^{11,12}$

In the current study, we demonstrated that the TDS value for 123I-BMIPP was significantly lower in patients with spontaneous anterograde flow than in those with total occlusion, suggesting beneficial effects of spontaneous anterograde flow on preserving myocardial fatty acid metabolism in patients with AMI. This effect of spontaneous anterograde flow also may contribute to the preservation of left ventricular function during the early phase of AMI.

The area at risk and the salvaged myocardium can be estimated accurately by myocardial perfusion imaging performed before and after reperfusion therapy ${ }^{13,14}$ Because this examination requires tracer administration on admission and imaging shortly after reperfusion therapy, it is unpopular and frequently avoided under serious conditions. Previous studies demonstrated that the uptake of 123I-BMIPP at 1 week was similar to the uptake of technetium-99m tetrofosmin before reperfusion therapy, and 123I-BMIPP has been used to identify the area at risk in the subacute phase of AMI 15 In the current study, there was no significant difference in the culprit lesion location between patients with spontaneous anterograde flow and those with total occlusion. However, the TDS value for ${ }^{123}$ I-BMIPP was significantly lower in patients with spontaneous anterograde flow than in those with total occlusion, suggesting that the ${ }^{123}$ IBMIPP SPECT image might underestimate the area at risk especially in patients with spontaneous anterograde flow.

Spontaneous Anterograde Flow vs Very Early Reperfusion

Brodie et al previously reported that time to reperfusion, up to $2 \mathrm{~h}$, was an important determinant of survival and recovery of left ventricular function, and that after $2 \mathrm{~h}$, recovery of left ventricular function was modest and survival was relatively independent of the time to reperfusion. 16 From this point of view, it is important to assess whether or not the beneficial effects of spontaneous anterograde flow 
were merely related to very early reperfusion.

In the current study, we demonstrated that the TDS values for ${ }^{201} \mathrm{Tl}$ and ${ }^{123} \mathrm{I}-\mathrm{BMIPP}$, which reflected myocardial perfusion and fatty acid metabolism respectively, were lower in patients with spontaneous anterograde flow than in those with very early reperfusion. In addition, we have previously reported that acute and predischarge LVEF are significantly better in patients with spontaneous anterograde flow than in those with very early reperfusion? Our current results suggest that the beneficial effects of spontaneous anterograde flow are related not only very early reperfusion but also to other mechanisms such as intermittent occlusion. On the other hand, in spontaneous anterograde flow, angiography was performed $4.3 \pm 3.1 \mathrm{~h}$ after the onset. Even in spontaneous anterograde flow, the TDS value for ${ }^{201} \mathrm{Tl}$ was significantly lower in the 19 patients in whom time to reperfusion was within $4 \mathrm{~h}$ than in the 9 patients in whom time to reperfusion was more than $4 \mathrm{~h}$, although the TDS value for ${ }^{123}$ I-BMIPP was similar for the 2 groups (data not shown). These results suggest that it is important for myocardial salvage to obtain persistent TIMI 3 flow with reperfusion therapy as early as possible even in patients with spontaneous anterograde flow.

\section{Study Limitations}

First, the allocation of reperfusion therapy was based on the physician's decision, but there was no significant difference in the choice of reperfusion therapy between patients with spontaneous anterograde flow and those with total occlusion, and final TIMI 3 flow was obtained in all patients. Second, the ${ }^{123}$ I-BMIPP SPECT image was obtained only once approximately 6 days after onset. In patients with spontaneous anterograde flow, especially fatty acid utilization may improve, to a certain degree, in the first few days after the onset of AMI. Although it is certain that spontaneous anterograde flow preserves myocardial fatty acid metabolism during the early phase of AMI, further studies are necessary to clarify the temporal process of myocardial fatty acid metabolism in patients with spontaneous anterograde flow or total occlusion. Third, this study could not demonstrate a significant difference in the TDS values for ${ }^{201} \mathrm{Tl}$ and ${ }^{123} \mathrm{I}$-BMIPP between patients with very early reperfusion and those with late reperfusion. Possible reasons are that patients with very early reperfusion had less prodromal angina ${ }^{17}$ and had the culprit lesion proximal to the first septal branch significantly more often than those with late reperfusion. Finally, predischarge angiography was performed in only $78 \%$ of the patients. It remains unclear whether the remaining patients had a patent infarct artery at the time of SPECT, but it should be noted that patients with signs of re-infarction were excluded from this study.

\section{Conclusion}

Spontaneous anterograde flow of the infarct artery preserved not only myocardial perfusion but also fatty acid metabolism in patients with anterior AMI. These beneficial effects of spontaneous anterograde flow are related not only to very early reperfusion but also to other mechanisms such as intermittent occlusion. ${ }^{123}$ I-BMIPP SPECT image may underestimate the area at risk, especially in patients with spontaneous anterograde flow.

\section{References}

1. Stone GW, Cox D, Garcia E, Brodie BR, Morice MC, Griffin J, et al. Normal flow (TIMI-3) before mechanical reperfusion therapy is an independent determinant of survival in acute myocardial infarction: Analysis from the primary angioplasty in myocardial infarction trials. Circulation 2001; 104: 636-641.

2. Lee CW, Hong MK, Lee JH, Yang HS, Kim JJ, Park SW, et al. Determinants and prognostic significance of spontaneous coronary recanalization in acute myocardial infarction. Am J Cardiol 2001; 87: $951-954$

3. Ishihara M, Inoue I, Kawagoe T, Shimatani Y, Kurisu S, Nishioka K, et al. Impact of spontaneous anterograde flow of the infarct artery on left ventricular function in patients with a first anterior wall acute myocardial infarction. Am J Cardiol 2002; 90: 5-9.

4. Tamaki N, Kawamoto M, Yonekura Y, Fujibayashi Y, Takahashi N, Konishi J, et al. Regional metabolic abnormality in relation to perfusion and wall motion in patients with myocardial infarction: Assessment with emission tomography using an iodinated branched fatty acid analog. J Nucl Med 1992; 33: 659-667.

5. Hashimoto A, Nakata T, Tsuchihashi K, Tanaka S, Fujimori K, Iimura O. Postischemic functional recovery and BMIPP uptake after primary percutaneous transluminal coronary angioplasty in acute myocardial infarction. Am J Cardiol 1996; 77: 25-30.

6. Ito T, Tanouchi J, Kato J, Morioka T, Nishino M, Iwai K, et al. Recovery of impaired left ventricular function in patients with acute myocardial infarction is predicted by the discordance in defect size on ${ }^{123}$ I-BMIPP and ${ }^{210}$ Tl SPET images. Eur J Nucl Med 1996; 23: 917-923.

7. The TIMI Study Group. The Thrombolysis in Myocardial Infarction (TIMI) trial. N Engl J Med 1985; 312: 932-936.

8. Kurisu S, Inoue I, Kawagoe T, Ishihara M, Shimatani Y, Nishioka K, et al. Myocardial perfusion and fatty acid metabolism in patients with tako-tsubo-like left ventricular dysfunction. J Am Coll Cardiol 2003; 41: 743-748.

9. Matsunari I, Saga T, Taki J, Akashi Y, Hirai J, Wakasugi T, et al. Kinetics of iodine-123-BMIPP in patients with prior myocardial infarction: Assessment with dynamic rest and stress images compared with stress thallium-201 SPECT. J Nucl Med 1994; 35: 1279-1285.

10. Kawamoto M, Tamaki N, Yonekura Y, Tadamura E, Fujibayashi Y, Magata Y, et al. Combined study with I-123 fatty acid and thallium201 to assess ischemic myocardium: Comparison with thallium redistribution and glucose metabolism. Ann Nucl Med 1994; 8: 47-54.

11. Tateno M, Tamaki N, Yukihiro M, Kudoh T, Hattori N, Tademura E, et al. Assessment of fatty acid uptake in ischemic heart disease without myocardial infarction. J Nucl Med 1996; 37: 1981-1985.

12. Takeishi Y, Sukekawa H, Saito H, Nishimura S, Shibu T, Sasaki Y, et al. Clinical significance of decreased myocardial uptake of 123-I BMIPP in patients with stable effort angina pectoris. Nucl Med Commun 1995; 16: $1002-1008$.

13. Gibbons RJ, Verani MS, Behrenbeck T, Pellikka PA, O'Connor MK, Mahmarian JJ, et al. Feasibility of tomographic 99mTc-hexakis-2methoxy-2-methylpropyl-isonitrile imaging for the assessment of myocardial area at risk and effect of treatment in acute myocardial infarction. Circulation 1989; 80: $1277-1286$.

14. Matsuo H, Watanabe S, Nishida Y, Matsubara T, Kano M, Sugiyama A, et al. Assessment of area at risk and efficacy of treatment in patients with acute coronary syndrome using $99 \mathrm{mTc}$ tetrofosmin imaging in humans. Ann Nucl Med 1993; 7: 231-238.

15. Kawai Y, Tsukamoto E, Nozaki Y, Kishino K, Kohya T, Tamaki N. Use of 123I-BMIPP single-photon emission tomography to estimate areas at risk following successful revascularization in patients with acute myocardial infarction. Eur J Nucl Med 1998; 25: 1390-1395.

16. Brodie BR, Stuckey TD, Wall TC, Kissling G, Hansen CJ, Muncy $\mathrm{DB}$, et al. Importance of time to reperfusion for 30-day and late survival and recovery of left ventricular function after primary angioplasty for acute myocardial infarction. J Am Coll Cardiol 1998; 32: $1312-1319$

17. Ishihara M, Sato H, Tateishi H, Kawagoe T, Shimatani Y, Kurisu S, et al. Implications of prodromal angina pectoris in anterior wall acute myocardial infarction: Acute angiographic findings and long-term prognosis. J Am Coll Cardiol 1997; 30: 970-975. 